\title{
Genomic Education - Bench to Bedside: A Novel Approach to Teaching Genetic Diagnosis
}

\author{
J. Keith Williams \\ Nova Southeastern University, Ft. Lauderdale, willjohn@nova.edu \\ Michael M. Segal \\ SimulConsult:MA, ijahsc@simulconsult.com \\ Lynn K. Feldman \\ SimulConsult: MA, feldman.lynn@simulconsult.com
}

Follow this and additional works at: https://nsuworks.nova.edu/ijahsp

Part of the Congenital, Hereditary, and Neonatal Diseases and Abnormalities Commons, Medical Education Commons, and the Medical Genetics Commons

\section{Recommended Citation}

Williams JK, Segal MM, Feldman LK. Genomic Education - Bench to Bedside: A Novel Approach to Teaching Genetic Diagnosis. The Internet Journal of Allied Health Sciences and Practice. 2021 Jan 01;19(2), Article 15.

This Concept Piece is brought to you for free and open access by the College of Health Care Sciences at NSUWorks. It has been accepted for inclusion in Internet Journal of Allied Health Sciences and Practice by an authorized editor of NSUWorks. For more information, please contact nsuworks@nova.edu. 


\title{
Genomic Education - Bench to Bedside: A Novel Approach to Teaching Genetic Diagnosis
}

\begin{abstract}
Problem: Teaching genetic diagnosis is required in all medical schools and physician assistant programs. However, with thousands of relevant findings and thousands more rare diseases, lectures and narrative resources are inadequate for the task. Whatever information that is taught is easily forgotten and does not carry over into the clinic. Many rare disease patients suffer through "diagnostic odysseys" (3 to 30 years to correct diagnosis). Approach: We used a commercially available diagnostic decision support system (DDSS) that encompasses all Mendelian disorders with known genes, together with other conditions in their differential diagnosis, and a case-based educational approach to teach diagnostic skills in a way that could then be replicated in the clinic. After a lecture, which included a demonstration using the DDSS with a sample case, 74 students were assigned to replicate the sample case at home and then complete 7 other anonymized cases, all with known rare diagnoses. After each case, students saved the "patient summary" that included the findings entered and differential diagnosis list and submitted it as homework. Students also completed a questionnaire about their experience, including satisfaction. Outcomes: Students were effective at diagnosing rare diseases in 483 of the 514 testing instances, a $94 \%$ success rate, with success defined as the correct diagnosis being listed in the differential diagnosis. Eighty-five percent of students rated this interactive learning session "highly," encouraged us to repeat the assignment next year, and $89 \%$ reported that they wanted to use the DDSS during their clinical rotations in the coming year. Next Steps: We plan to refine the cases, add more material on findings, and ensure that all the synonyms students might use are in the software tool. We plan to repeat the program next year and recommend its use more widely in medical education.
\end{abstract}

\section{Author Bio(s)}

J. Keith Williams, MPAS, PA-C Emeritus, is an Assistant Professor in the Physician Assistant Program at Nova Southeastern University in Ft. Lauderdale, FL.

Michael M. Segal, MD, PhD, is a neurologist and neuroscientist who was the key author of the diagnostic decision support software and a major contributor to its database.

Lynn K. Feldman, MBA, CEO, of SimulConsult.

\section{Acknowledgements}

Acknowledgements: None Funding/Support: Not applicable Other Disclosures: MMS and LKF have ownership stakes in SimulConsult Ethical Approval: Exempt 2020-455-NSU Disclaimers: None Previous Presentations: Not applicable 


\title{
1IVAHSP
}

\section{The Internet Journal of Allied Health Sciences and Practice \\ Dedicated to allied health professional practice and education}

Vol. 19 No. 2 ISSN 1540-580X

\section{Genomic Education - Bench to Bedside: A Novel Approach to Teaching Genetic Diagnosis}

\author{
J. Keith Williams ${ }^{1}$ \\ Michael M. Segal2 \\ Lynn K. Feldman² \\ Nova Southeastern University \\ SimulConsult: MA \\ United States
}

\begin{abstract}
Problem: Teaching genetic diagnosis is required in all medical schools and physician assistant programs. However, with thousands of relevant findings and thousands more rare diseases, lectures and narrative resources are inadequate for the task. Whatever information that is taught is easily forgotten and does not carry over into the clinic. Many rare disease patients suffer through "diagnostic odysseys" (3 to 30 years to correct diagnosis). Approach: We used a commercially available diagnostic decision support system (DDSS) that encompasses all Mendelian disorders with known genes, together with other conditions in their differential diagnosis, and a case-based educational approach to teach diagnostic skills in a way that could then be replicated in the clinic. After a lecture, which included a demonstration using the DDSS with a sample case, 74 students were assigned to replicate the sample case at home and then complete 7 other anonymized cases, all with known rare diagnoses. After each case, students saved the "patient summary" that included the findings entered and differential diagnosis list and submitted it as homework. Students also completed a questionnaire about their experience, including satisfaction. Outcomes: Students were effective at diagnosing rare diseases in 483 of the 514 testing instances, a 94\% success rate, with success defined as the correct diagnosis being listed in the differential diagnosis. Eighty-five percent of students rated this interactive learning session "highly," encouraged us to repeat the assignment next year, and $89 \%$ reported that they wanted to use the DDSS during their clinical rotations in the coming year. Next Steps: We plan to refine the cases, add more material on findings, and ensure that all the synonyms students might use are in the software tool. We plan to repeat the program next year and recommend its use more widely in medical education.
\end{abstract}

Keywords: medical education, genetic diagnosis, rare diseases, diagnostic odyssey, case-based learning (CBL), health information systems, Diagnostic Decision Support System (DDSS), clinical decision support. 


\section{STATEMENT OF THE PROBLEM}

Diagnostic Decision Support Systems (DDSSs) are a key type of clinical decision support needed in genomics to supplement a shortage of trained clinicians to deal with the inherent complexity of genetic diagnosis and help end diagnostic odysseys. Complexity arises from the multi-system nature of genetic diseases, the variable expression in patients, and the degree of overlap in findings (i.e., signs, symptoms, and test results) among genetic conditions, sometimes differentiated mainly by "onset age" of individual findings. While such tools are increasingly used in the clinic, they have not been widely used in medical education.

Healthcare education now requires competencies in genomic medicine for Physicians, Physician Assistants, Pharmacists, Genetic Counselors, and Nurses. ${ }^{1}$ However, the number of rare findings and ultra-rare conditions makes it difficult to teach students about genetic diagnosis in a manner that carries over into clinical practice.

Rare diseases, particularly genetic conditions, defy ready diagnosis. While such diseases are individually rare, they are so numerous as to be collectively present in $8 \%$ of the population, making genetics a crucial part of medical education. ${ }^{2}$ Moreover, medical research has been adding hundreds of new diseases annually. The proliferation of individual diseases, each with an average of 40 findings, many present in only a subset of the patients with the condition, overwhelms the ability of a clinician to keep and sort the information in their head, let alone a student doing so. ${ }^{3}$

Today the medical system copes by supporting ever greater specialization and referrals, but that is costly and does not work well. At least $25 \%$ of rare disease patients suffer through "diagnostic odysseys" (3 to 30 years to correct diagnosis), including frequent misdiagnoses ( $40 \%$ of patients), and inappropriate care (16\% had futile surgeries, $33 \%$ had other incorrect treatments). ${ }^{4}$

\section{APPROACH}

Our overall approach was to use an interactive DDSS, the SimulConsult Phenome Analyzer, to make clear the iterative nature of differential diagnosis, especially in complex disorders. ${ }^{5-7}$ Many ways in which expert clinicians mull complex diagnostic cases has a parallel in the software, and these features were demonstrated to the students. The main screen is shown in Figure 1, for an 8month-old boy after entry of two positive findings (kyphosis and macrocephaly, both with an onset at 6-months-old) and one negative finding (absence of short stature). The display shows a differential diagnosis with "MPS I, severe: Hurler syndrome classic" ranked \#1 in probability and further findings likely to be useful, with "Corneal clouding or opacity" ranked \#1 in usefulness in distinguishing among the diseases in the differential diagnosis.

\section{Using a DDSS to Make Explicit the Steps in Doing Complex Differential Diagnosis}

During the lecture, we walked through a case example to consider the importance of onset, pertinent negatives, cost, reliability of test results in prioritizing testing, and the fit with the disease. The most fundamental task - figuring out which other findings would be useful in the context of the evolving differential diagnosis (i.e., ranked list of diseases the patient might have) - was emphasized. This included a demonstration of how pertinent negatives can clear the field of similar diseases without a particular finding or with a particular finding and a different onset.8,9 A teacher's guide helps the lecturer efficiently emphasize each of these points at a useful time in the progression but leaves the lecturer free to answer questions and proceed in a different sequence.

\section{Creating Simulated Cases for User Testing}

SimulConsult provided the demonstration case and 7 other teaching cases, all based on real patients, but anonymized. Each case was summarized in a short vignette (average 138 words, range 58 to 271). All the case vignettes had been validated previously in other research with generalist and specialist physicians. ${ }^{6}$

\section{Population}

Seventy-four students ( $n=74$ ) registered to use the software in their fourth and last semester of didactic coursework (15 mo.) in the Physician Assistant program before starting on clinical rotations $(12 \mathrm{mo}$.) in their second year of training.

\section{Process}

The students were given 8 cases with test-proven diagnoses: the case from the lecture for which the diagnosis was revealed and 7 cases for which the diagnoses were not revealed. To familiarize themselves with the software, each student was encouraged to start by replicating the demonstration case used in the lecture, for which they knew the answer. They also had a 3-page guide to all the features, which they were encouraged to explore.

After entering all the case findings, they were asked to save the patient summary PDF file, which could be generated by the software (see Figure 2, shown for the demonstration case). The PDF file includes the name of the clinician, as they might give the 
summary to a patient. The students saved the PDF files and submitted them as homework via the homework platform Canvas. After completing the cases, students were asked to complete an optional online satisfaction survey anonymously.

\begin{tabular}{|c|c|c|c|c|c|}
\hline \multicolumn{2}{|c|}{ Differential } & Add findings & Add tests & Phenotype 21 & \\
\hline \multicolumn{3}{|c|}{ MPS I, severe: Hurler syndro... } & Presence $\mathbf{i}$ & \multicolumn{2}{|l|}{ Findings ranked by usefulness $\mathrm{i}$} \\
\hline \multicolumn{3}{|c|}{ Hypomelanosis of Ito } & $?=$ & \multicolumn{2}{|l|}{ Corneal clouding or opacity } \\
\hline \multirow{2}{*}{\multicolumn{3}{|c|}{ Acromesomelic dysplasia, M... }} & $?$ & \multicolumn{2}{|l|}{ Respiratory difficulty } \\
\hline & & & & \multirow{2}{*}{\multicolumn{2}{|c|}{ Mouth: tonsils large }} \\
\hline \multicolumn{3}{|c|}{ NF1: Neurofibromatosis 1} & $?=$ & & \\
\hline \multicolumn{3}{|c|}{ GM1 gangliosidosis, type I (i... } & $? \cdot$ & \multicolumn{2}{|l|}{ Eyebrows thick } \\
\hline \multicolumn{3}{|c|}{ MASA: mental retardation, a... } & $?=$ & \multicolumn{2}{|l|}{ Weight low or weight loss } \\
\hline \multicolumn{3}{|c|}{ MPS VII: Sly syndrome, infan... } & $?=$ & \multicolumn{2}{|l|}{ Face, coarse features } \\
\hline \multicolumn{3}{|c|}{ Weaver syndrome } & $?=$ & \multicolumn{2}{|l|}{ Hypotonia } \\
\hline \multicolumn{3}{|c|}{ Osteopathia striata with cra... } & $?=$ & \multicolumn{2}{|l|}{ Mouth: alveolar ridge overgrowth } \\
\hline \multicolumn{3}{|c|}{ Macrocephaly, benign familial } & $?=$ & \multicolumn{2}{|l|}{ Skin: hypopigmented lesions } \\
\hline \multicolumn{3}{|c|}{ Glass syndrome } & $?=$ & \multicolumn{2}{|l|}{ Peripheral nerve entrapment or compression } \\
\hline \multicolumn{3}{|c|}{ Schimmelpenning-Feuerstei... } & $?=$ & \multicolumn{2}{|l|}{ Skin: areas or lines of pigment or redness } \\
\hline \multicolumn{3}{|c|}{ Sialidosis II, infantile } & $?=$ & \multicolumn{2}{|l|}{ Frontal bossing } \\
\hline \multicolumn{3}{|c|}{ NS1: Noonan syndrome, PTP... } & $?=$ & \multicolumn{2}{|l|}{ Mouth: tongue large } \\
\hline \multicolumn{3}{|c|}{ MRXSC: mental retardation, ... } & $?=$ & \multicolumn{2}{|l|}{ Epicanthus } \\
\hline \multirow{2}{*}{ Top } & $<$ & $1>$ & $?=$ & \multicolumn{2}{|l|}{ Infections, frequent } \\
\hline & & & Top & 1 of all findings & Highest usefulne \\
\hline
\end{tabular}

Figure 1. Main Diagnosis Screen of the SimulConsult $₫$ Phenome Analyzer 


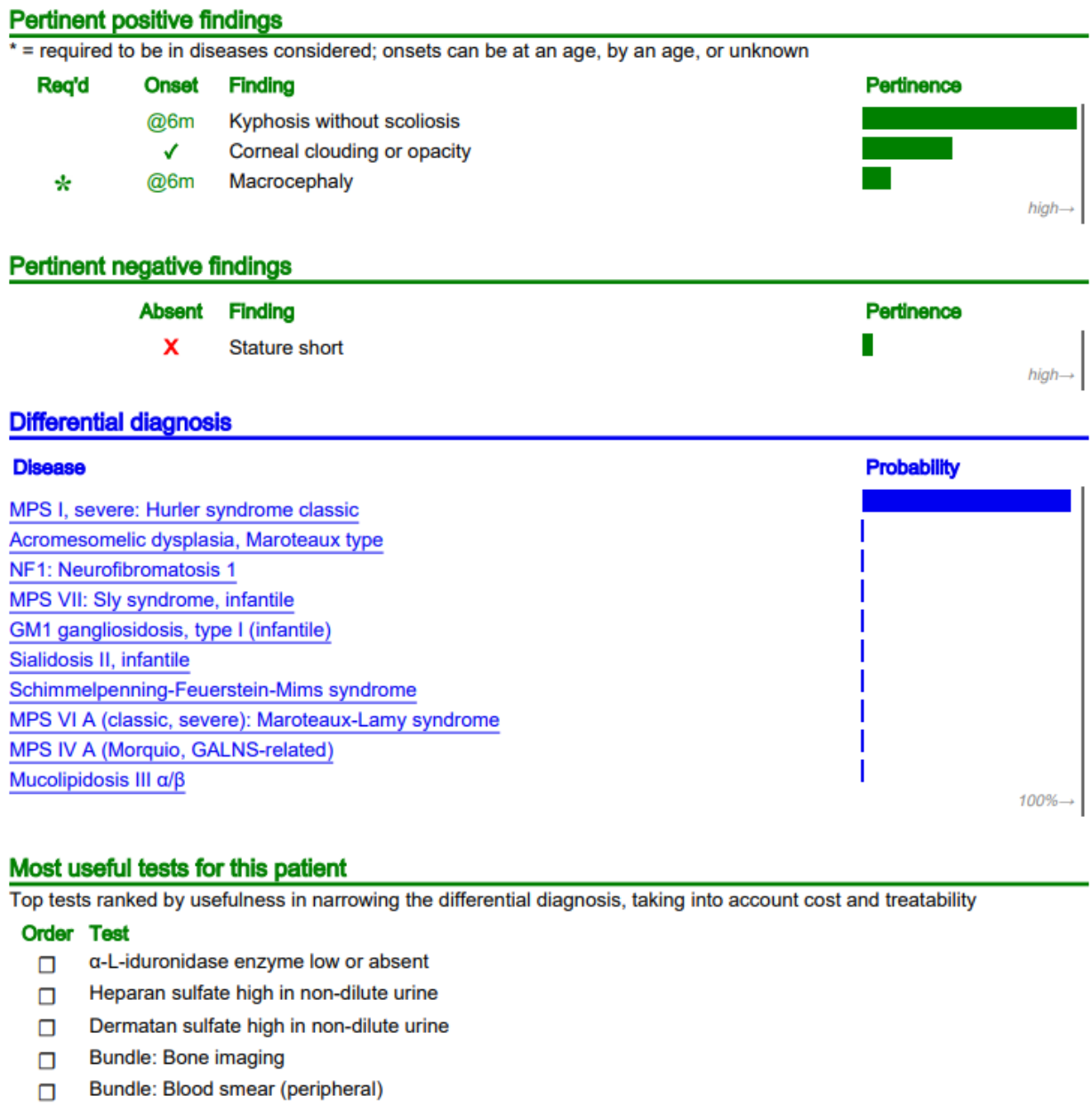

Generated by SimulConsult 8 on 29 July 2020 19:56 using interface of 2020 July 28 14:04, algorithms of 2020 July 24 20:00 and database of 29 July 2020 19:16. All times are UTC. Care setting was primary. Onset was used.

Figure 2. Patient Summary

Overall, 48 students had no diagnostic errors (65\%), 21 students (28\%) had 1 error, and 5 students $(7 \%)$ had 2 errors as seen in Figure 3. For the 483 testing instances without diagnostic errors, the average rank of the ultimate diagnosis in the differential diagnosis was $1.9( \pm 0.8)$, where a rank of 1.0 is the best score, (ranks by case: 1.2, 2.6, 1.6, 1.3,1.3, 2.3, and 3.3). With such a high rank, the ultimate diagnosis is likely to be actively considered, resulting in testing to confirm or exclude such a diagnosis.

Most errors arose from the difficulties that students had translating the case vignette description to findings in the decision support. For example, a case vignette description of "rhythmic head twitching" mapped to the finding "stereotypies." "Mild asterixis" mapped to "tremor." Translating the descriptions proved difficult for some students, despite the search functionality in the software using synonyms that resulted in the correct findings being displayed in some of these instances in tooltips that could be explored to see the synonyms. Another source of diagnostic error was that some students added only clinical findings and neglected to add important laboratory results described in the case vignette, something that can be emphasized in future presentations. Other sources of diagnostic error included leaving out pertinent negatives or adding ones not mentioned in the case, not including family history, and adding a previous misdiagnosis as a positive finding. This highlights the need for greater assistance in helping students learn about the approximately 1,200 clinical findings relevant in genetics. Developing a companion training module focusing on unusual findings, is something we plan to do next year. We will also emphasize in the lecture the benefit of the "Tips" section of the software and the ability to hover over a finding to view synonyms. 


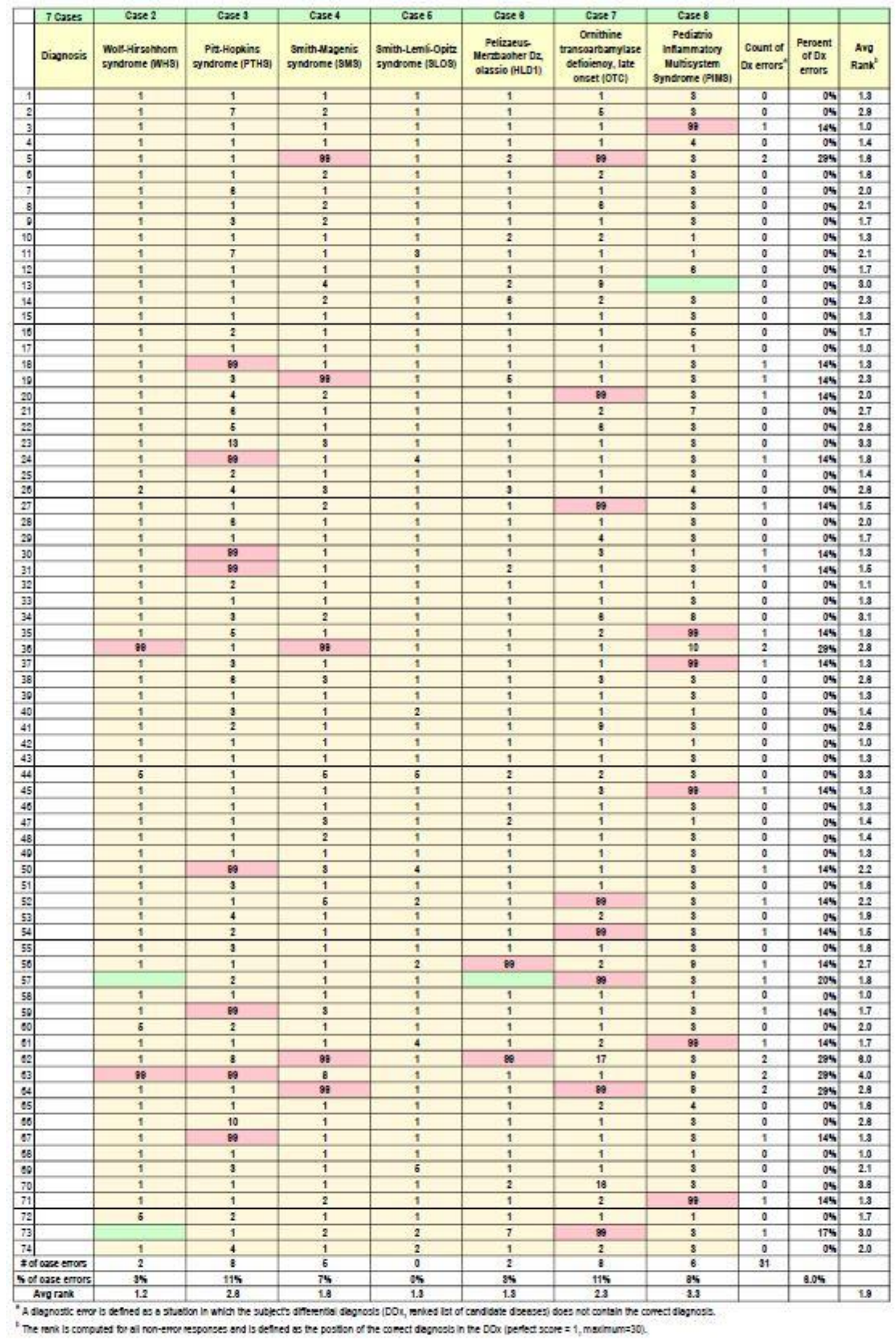

Figure 3. PA Students Data of 7 Case Vignettes 
In a previous study with physician subjects using multiple vignettes, they were asked for a differential diagnosis before and after using the SimulConsult software. ${ }^{6}$ Six of the seven vignettes used in the Physician Assistant (PA) study were ones used in the physician study and were used to compare the results. The PA students were not asked for a differential diagnosis before using the software because those topics had not been covered yet. So, only after using the software results were used in the comparison Figure 4.

\begin{tabular}{|c|c|c|c|c|c|c|c|c|}
\hline Metric & Cohort & $\begin{array}{l}\text { Wolf-Hirschhorn } \\
\text { syndrome (WHS) }\end{array}$ & $\begin{array}{c}\text { Pitt-Hopkins } \\
\text { syndrome (PTHS) }\end{array}$ & $\begin{array}{l}\text { Smith-Magenis } \\
\text { syndrome (SMS) }\end{array}$ & $\begin{array}{l}\text { Smith-Lemli- } \\
\text { Opitz syndrome } \\
\text { (SLOS) }\end{array}$ & $\begin{array}{c}\text { Pelizaeus- } \\
\text { Merzbacher Dz, } \\
\text { classic (HLD1) }\end{array}$ & $\begin{array}{c}\text { Ornithine } \\
\text { transcarbamylase } \\
\text { deficiency, late } \\
\text { onset (OTC) } \\
\end{array}$ & $\begin{array}{c}\text { Average } \\
\text { score }\end{array}$ \\
\hline \multirow{3}{*}{ Error' } & 5 Senior MDs & $0.0 \%$ & $80.0 \%$ & $40.0 \%$ & $0.0 \%$ & $17.0 \%$ & $0.0 \%$ & $22.8 \%$ \\
\hline & 11 Junior MDs & $0.0 \%$ & $45.0 \%$ & $9.0 \%$ & $0.0 \%$ & $0.0 \%$ & $22.0 \%$ & $12.7 \%$ \\
\hline & 74 PA students & $2.8 \%$ & $10.8 \%$ & $6.8 \%$ & $0.0 \%$ & $2.7 \%$ & $10.8 \%$ & $5.6 \%$ \\
\hline \multirow{3}{*}{ Rank $^{b}$} & 5 Senior MDs & 1.5 & 1.0 & 1.0 & 1.0 & 1.2 & 2.0 & 1.3 \\
\hline & 11 Junior MDs & 1.0 & 1.7 & 1.5 & 1.0 & 1.3 & 27 & 1.5 \\
\hline & 74 PA students & 1.2 & 2.6 & 1.6 & 1.3 & 1.3 & 2.3 & 1.7 \\
\hline
\end{tabular}

'A diagnosbic error is defined as a situabion in which the subject's differental diagnosis (ranked list of candidale diseases) does not contain the correct diagnosis s The rank is computed for all non-error responses and is defined as, where the ulimale diagnosis is ranked on the list of candidate diseases (perfect score = 1) Figure 4. MD \& PA Comparison Table of 6 Vignettes using SimulConsult Software

When comparing only the 6 case vignettes, the PA students error rate of $5.6 \%$ compared favorably to the generalist and specialist attending and resident physicians weighted average of $15.9 \%$. Though improvements in the software and database since the physicians were tested (7 years) could have been a factor in the improved performance of the physician assistant students. ${ }^{6}$ This is evidence confirming the hypothesis that training students in the use of the diagnostic decision support system can be an effective, systematic way to help trainees improve their genetic diagnostic success early in their careers. Nevertheless, we considered the ability of the PA students to do as well as the physicians to be remarkable, and an argument for training PAs to help in the diagnosis process using such software.

\section{SimulConsult Satisfaction Survey Results}

In general, students are trained to be specific as when writing a case note, such as a rash on the left lower extremity. Using the software, they were often uncomfortable working at a less granular level such as adding just the type of rash without the location; they had too little knowledge of the conditions in the differential diagnosis to know if all the detail such as localization was relevant. One student commented, "It was a little difficult knowing which words to use (when searching) for the specific findings outlined within some cases. If I was unable to find a finding that made sense, I left it out - which may have affected the diagnosis reached." Students thought it would be helpful to increase their familiarity with key terms and their synonyms. Next year, we will also explicitly teach the students the process of putting in the known findings and then iteratively seeing which other findings are deemed useful by the software - since the suggested findings based on usefulness in narrowing the differential diagnosis may zero in on the finding that the student is having trouble assessing.

Most of the students (57 of 74 or $77 \%$ ) completed the satisfaction survey. It revealed that none were aware of the DDSS's existence prior to the class. They reported that they spent an average of 6.3 minutes per case $( \pm 2.2$ minutes), slightly less than more experienced clinicians, who averaged 7 to 9 minutes in the two previously referenced studies using an older version of the software.., 6

Most respondents, 54 of $57(95 \%)$ reported they found all the screens and features they were seeking. Two reported difficulty finding the family history, and one expected to be able to comment that a sibling was not merely affected but had died (not currently supported). One had difficulty finding a test result.

Forty-one of 57 students $(72 \%)$ reported that the software was "easy" or "very easy" to use. Fourteen students (25\%) found it adequately easy to use, and 2 students (4\%) found it "somewhat hard." Students commented that the DDSS "was very clear and easy to navigate!" 
Most students, 55 of $57(96 \%)$, reported that the visualization of the differential diagnosis was helpful, and one student commented that "The bars showing the frequency were helpful to see." They also liked "being able to save several patients, so if you have to add information you can go back to the previous case without needing to start from the beginning." Nearly all students, 56 of 57 $(98 \%)$ reported that the process of working through a case helped illustrate the iterative nature of diagnosis, and the various approaches used by clinician to develop and refine a differential diagnosis. Students rated their satisfaction with the tool on a 0 to 100 scale as $85 \%$. Most students (89\%) reported that they would like to be able to use SimulConsult in their clinical work. Their comments were positive, such as "SimulConsult is a great tool to have as a healthcare provider!"; "Very interesting tool to know of and to know how to use for any clinician in any field in my opinion"; and "Loved the software."

Students also liked the case method, rating it $85 \%$ on a 0 to 100 scale. One student commented "I believe that the assignment was very interesting and helpful, as it made us aware of another useful tool we can utilize in clinical practice. The software was very easy to navigate, and I would definitely recommend keeping this assignment for next year's class." They reported learning a lot, "I really enjoyed the assignment and would be glad to do it again! Great experience. Learned a lot of new diagnoses and signs!" And they viewed the case-based approach as a success, reporting "I really enjoyed this - very engaging and exciting!"

\section{NEXT STEPS}

This study reports on the innovative use of a diagnostic decision support tool that covers $>7,400$ diseases, including all Mendelian conditions with known genes, to teach genetic diagnosis. The initial use was a success and will be repeated in the following year. To improve on the experience reported here, we plan to clarify the description of findings that caused problems, and to supplement with core findings, that should help students engage more easily with the genetic cases. The finding-driven approach encouraged the students to think, not to fill in some form mindlessly, and helped improve their general skills in differential diagnosis. As a result, the approach should enable students to use and grow these skills during their clinical rotations. We also made the tool available to the students for their clinical year and will follow the adoption rate and level of use. Other PA programs and medical schools should consider utilizing the SimulConsult Phenome Analyzer in their genetic curriculum to help future clinicians eliminate the diagnostic odyssey of their patients with rare diseases.

Interestingly, PA case 8 was not included in the physician study because it was a COVID-19 vignette meant to mimic Kawasaki disease. After submitting this paper, a senior pediatric rheumatologist told us that half of their patients with Kawasaki disease now test positive for COVID-19. Accordingly, the distinction between parainfectious COVID-19 and Kawasaki disease, for which many PAs were marked down on in case 8 (the case with the lowest diagnostic rank, 3.3), is no longer thought to be a valid distinction. Therefore, the DDSS database will be changed to reflect that new knowledge and our choice of this case illustrated a diagnostic challenge that resulted in a new understanding of these diagnoses.

\section{REFERENCES}

1. Competency Map. Genetics/Genomics Competency Center. https://genomicseducation.net/competency. Published 2020. Accessed August 9, 2020.

2. Genetic and Rare Diseases Information Center (GARD). FAQs About Rare Diseases. https://rarediseases.info.nih.gov/diseases/pages/31/faqs-about-rare-diseases. Accessed August 6, 2020.

3. Segal MM. How doctors think, and how software can help avoid cognitive errors in diagnosis. Acta Paediatrica 2007; 96 : $1720-2$

4. Kole, A, Faurisson, F, Eurodis: The Voice of 12,000 Patients, Experiences and Expectations of Rare Disease Patients on Diagnosis and Care in Europe, A report based on the EurordisCare2 and EurordisCare3 Surveys, 2009

5. Segal MM, Athreya B, Son MB, Tirosh I, Hausmann JS, Ang EY, Zurakowski D, Feldman LK, Sundel RP. Evidence-based decision support for pediatric rheumatology reduces diagnostic errors. Pediatr Rheumatol Online J. 2016 Dec 13;14(1):67. doi: 10.1186/s12969-016-0127-z. PMID: 27964737; PMCID: PMC5155385.

6. Segal MM, Williams MS, Gropman AL, et al. Evidence-based decision support for neurological diagnosis reduces errors and unnecessary workup. J Child Neurol. 2014;29(4):487-492. doi:10.1177/0883073813483365

7. Segal, M.M., George, R., Waltman, P. et al. Clinician-centric diagnosis of rare genetic diseases: performance of a gene pertinence metric in decision support for clinicians. Orphanet J Rare Dis 15, 191 (2020). https://doi.org/10.1186/s13023$\underline{020-01461-1}$

8. Eddy, D.M. and C.H. Clanton, the art of diagnosis: solving the clinicopathological exercise. N Engl J Med, 1982. 306(21): p. 1263-8

9. Kassirer, J.P., Teaching clinical medicine by iterative hypothesis testing. Let's preach what we practice. N Engl J Med, 1983. 309(15): p. 921-3 